\title{
Disease and Diagnosis
}

\section{Generated Immune Responses and Antibody-Mediated Immunity Against Corona Virus Disease-19: An Updated Review}

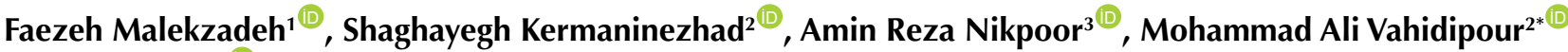 \\ , Ali Salimi As| $\mathbf{1}^{*} \mathbb{0}$ \\ ${ }^{1}$ Student Research Committee, Faculty of Para-Medicine, Hormozgan University of Medical Sciences, Bandar Abbas, Iran. \\ ${ }^{2}$ Student Research Committee, Faculty of Medicine, Hormozgan University of Medical Sciences, Bandar Abbas, Iran. \\ ${ }^{3}$ Department of Immunology, Faculty of Medicine, Hormozgan University of Medical Science, Bandar Abbas, Iran.
}

\begin{abstract}
Coronavirus disease 2019 (COVID-19) is a new pathogen which has become an uncontrollable pandemic. On the other hand, the humoral immune system, as a defensive line, protects the body by producing antibodies. Immunoglobulin M (IgM) and G (IgG) are considered as the most important antibodies that are produced by the immune system of the host. Previous evaluations on the production of $\lg M$ and $\operatorname{lgG}$ antibodies by patients suffering from severe acute respiratory syndrome coronavirus 2 (SARS-CoV-2) infection specifies that IgM is an acute phase antibody and increases on the first days of infection. Then, as the chronic phase begins, the level of the IgM decreases and switches to the production of IgG. In addition, the durability of the IgG antibody shows the duration of the immunity against COVID-19. Thus, the present review article focused on the antibody production mechanism in studies conducted on COVID-19 patients, the protection role of antibodies, and their stability.

Keywords: Humoral immune, Antibody, Seropositive, COVID-19, SARS-CoV-2
\end{abstract}

*Correspondence to Mohammad Ali Vahidipour, Ali Salimi Asl, Student Research Committee, Faculty of Medicine, Hormozgan University of Medical Sciences, Bandar Abbas, Iran. Tel:+989356911283; Email: mali_vahidipour@ yahoo.com:

Tel:+989307424323 Email: shervin7717@gmail. com

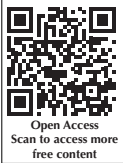

Received: July 22, 2020, Accepted: August 18, 2020, ePublished: December 30, 2020

\section{Introduction}

The novel coronavirus caused by severe acute respiratory syndrome coronavirus 2 (SARS-CoV-2) infection, which belongs to the beta-coronavirus genus of zoonotic origin as a common disease of humans and animals and has begun in Wuhan, China, has led to a widespread epidemic worldwide. This newly emerging disease has various mild to severe consequences (1). By binding to the angiotensin-converting enzyme 2 (ACE2) receptor, the virus enters the respiratory system through airborne particles and is directed into the respiratory tract (airways). if no proper immune response is generated, the virus can infect the alveoli and cause an infection. ACE2 is widely expressed in other tissues in addition to the respiratory system (2). The humoral immune system, which functions by producing antibodies to block and neutralize the virus, is stimulated when the coronavirus enters the host cell. Further, the period of antibody detection varies between 1 and 39 day(s) and the strength of the antibody response differs from one person to another, depending on several factors (3). The emergence of symptoms is associated with the increased sensitivity of serological tests compared to molecular tests (4). Given that the viral load varies at different stages of the disease, the viral ribonucleic acid (RNA) test is likely to provide a false-negative result at the beginning of the disease and, as a result, these individuals can spread the disease due to being a carrier (5). Therefore, serological tests can probably identify suspected individuals by measuring antibody responses and thereby control the spread of infection. Furthermore, antibodies will likely remain positive when patients test negative for the virus, leading to immunity against re-infection and the development of a vaccine for dealing with the virus. Given that the outbreak of the virus still exists, one cannot speak with certainty about the duration of immune responses after patients test negative for the virus. According to data from coronavirus disease 2019 (COVID-19) studies, this study aimed to fill the knowledge gap in the human immune response to SARS-CoV-2 infection, which may contribute to identifying individuals with suspected COVID-19 to control the prevalence of the disease and

(C) 2020 The Author(s). This is an open access article distributed under the terms of the Creative Commons Attribution License (http:// creativecommons.org/licenses/by/4.0/), which permits unrestricted use, distribution, and reproduction in any medium, provided the original work is properly cited. 
design an appropriate immunological intervention for the treatment, diagnosis, and development of prophylactic and therapeutic vaccines against COVID-19.

\section{About COVID-19}

SARS-CoV-2 is a human coronavirus that causes zoonotic diseases and presents a major threat to public health. Additionally, this infection can produce a range of different consequences. Approximately 1.2\%, 80.9\%, $13.8 \%, 4.7 \%$, and $6 \%$ were reported for asymptomatic infected individuals, as well as mild to moderate, severe, critical cases, and death, respectively. According to (6), COVID-19 is considered a self-limiting infectious disease, and most mild cases improve in 1-2 week(s).

Form December 31, 2019 when the first official case of COVID-19 reported by the World Health Organization till now (26 June 2020), 9473214 cases of COVID-19 with 484249 deaths have been reported in more than 210 countries worldwide and this rate is still rising. Although the mortality rate of SARS-CoV-2 is lower than that of SARS-CoV and Middle East respiratory syndrome coronavirus (MERS-CoV), the number of relative infection cases is more than 10 times higher than that of the others. Nonetheless, the exact route to the first case of infection is unknown (1). Moreover, in the early stages of the COVID-19 outbreak, the detected cases outside China were mostly passengers who got infected in China and then traveled to other areas. The compiled reports indicate that SARS-CoV-2 can also be transmitted from asymptomatic individuals or those with a mild infection. This feature may explain the sudden outbreak of the virus epidemic. Respiratory droplets and contact are the main routes of transmission. All races and ages are at risk of developing the disease. Nevertheless, the elderly, pregnant women and people with major disorders such as asthma, diabetes, cardiovascular disease, and cancer may be more susceptible to COVID-19, and smoking and obesity are among other underlying factors in this respect. In addition, individuals who have close contact with patients (their families and health care staff) or those with clinical symptoms constitute one part of the high-risk population (6).

\section{The Immune Response Against COVID-19}

During virus infection, host factors trigger an immune response against the virus. However, it is noteworthy that immunopathogenesis is associated with an immune response out of control, which may lead to pulmonary tissue damage, functional impairment, and reduced lung capacity. Chemotactic factors are critical for the immune responses against virus infections thus spectral changes in chemotactic factors may result in severely maladjusted immune responses. Further, immune insufficiency or misdirection may increase viral replication and inflict tissue damages while overactive immune responses may induce immunopathological conditions (7).

\section{Innate Immune Response}

The host innate immune system can detect viral infections by using pattern recognition receptors (PRRs). Currently, the known PRRs mainly include Toll-like receptors (TLRs), retinoic acid-inducible gene-I-like receptors, nucleotidebinding oligomerization domain-like receptors (NLRs), C-type lectin-like receptors, and free-molecule receptors in the cytoplasm, including cyclic GMP-AMP synthase (cGAS), IFN gamma inducible protein 16, the stimulator of the IFN gene, the DNA-dependent activator of the IFN-regulatory factor, and the like (7).

When the virus invades the host, first, PRRs recognize the viral nucleic acid and then collect the specific signal adapter protein, activate IFN regulatory factors 3 and 7 (IRF3 \& IRF7) before being translocated into the nucleus, and finally, promote the synthesis of type I IFNs. Subsequently, type I IFNs activate the downstream Janus kinase-signal transducer and the activator of the transcription signal pathway, which promotes the expression of IFN-stimulated genes. IFNs, as the major antiviral molecules of the host, limit the spread of the virus, and play an immunomodulatory role in promoting the macrophage phagocytosis of antigens and restricting natural killer cells to infected target cells and T/B cells. Therefore, blocking the production of IFNs exerts a direct effect on the survival of the virus in the host (7).

The activation of the complement system occurs in patients infected with MERS-CoV, SARS-CoV, and SARS-CoV-2 and appears to be an influential factor that has a destructive role in the pathogenesis of these viruses, especially in the development of acute respiratory distress syndrome and acute lung injury (8). C3a, C4a, and C5a, which are the components of the complement system, are part of the innate immune system of the body thus their response is nonspecific and immediate. Furthermore, these complement components with anaphylatoxin and proinflammatory properties can exacerbate viral infection and acute lung injury (9).

\section{Acquired Immune System Response to COVID-19 T Cell Response in COVID-19}

MERS-CoV and SARS-CoV are beta-coronaviruses that can cause lower respiratory tract infections and extrapulmonary manifestations. In this regard, $\mathrm{T}$ cells, $\mathrm{CD}^{+} \mathrm{T}$ cells, and particularly $\mathrm{CD}^{+} \mathrm{T}$ cells play an important antiviral role in balancing the combat against pathogens and the risk of developing autoimmunity or overwhelming inflammation. Moreover, $\mathrm{CD}^{+} \mathrm{T}$ cells can promote the production of virus-specific antibodies by activating T-dependent $\mathrm{B}$ cells. However, $\mathrm{CD}^{+} \mathrm{T}$ cells are cytotoxic and can destroy virus-infected cells. Similarly, $\mathrm{CD}^{+} \mathrm{T}$ cells account for about $80 \%$ of total inflammatory cells in the pulmonary interstitium in SARS-CoVinfected patients and play a significant role in clearing infected cells and inflicting immune injury (7). Some of the produced vaccines are based on the activation of 
$\mathrm{CD}^{+}$cytotoxic T lymphocytes (CTLs). More precisely, they activate CD8 ${ }^{+}$CTLs solely or indirectly by activating $\mathrm{CD}^{+} \mathrm{T}$ helper cells. For an effective and strong induction of the CTL response that would lead to tumor inhibition, the antigen should be presented to $\mathrm{CD} 8^{+} \mathrm{CTL}$ via major histocompatibility complex class I (10-12).

\section{B Cell Response in COVID-19}

The humoral immune response is critical for clearing the body of pathogenic viruses and mainly consists of memory responses that often prevent re-infection. The $\mathrm{B}$ cell response to a virus is useful for protecting against the initial challenge and providing widespread immunity against re-infection. After being infected, plasma cells, which are formed in acute and recovery phases, continue to secrete antibodies. By producing new plasma cells, memory $\mathrm{B}$ cells can respond with greater intensity and speed in re-infection with the virus. Long-term protection in disease is achieved through the induction of memory plasma cells and memory B cells. Given that not a long time has passed since the novel coronavirus outbreak, one cannot comment on the nature of the extent of long-term immune responses (13).

It should be noted that memory and plasma cells play an important role in controlling the virus, increasing immune compatibility, and inducing specific antibodies. Several genes associated with $\mathrm{B}$ cell activation were primarily expressed in the plasma cells of patients in the early stages of treatment (the early stages (ERS) group) as compared to healthy controls. These genes included S100A8 (calcium- and zinc-binding protein), immunoglobulin lambda-like polypeptide 5 (IGLL5), signal sequence receptor subunit 3 (SSR3), immunoglobulin heavy constant alpha 1 (IGHA1), X-box binding protein 1 (XBP1), and marginal zone $B$ and $B_{1}$ cell specific-protein (MZB1). Gene ontology analysis showed that the XBP1, IGHA1, polymerase RNA II SUBUNIT L (POLR2L), JUN, MZB1, and zinc finger protein 36 genes were overexpressed in plasma cells. This increase in the expression of $\mathrm{B}$ cells indicates viral replication and transcription in patients with COVID-19. Using single-cell B-cell receptor sequencing (SC-BCR-Seq) to assess the status of $\mathrm{B}$-cell clonal expression in the blood of patients, it was revealed that the naïve $\mathrm{B}$ cell demonstrated slight clonal expansion of the interleukin 4 receptor (IL4R) whereas memory B-cells represented high levels of $\mathrm{CD} 27^{+}$and $\mathrm{CD} 38^{+}$. There were more B-cell clones in patients in the ERS group (early recovery, it took less than 7 days to test negative for the viral nucleic acid) as compared to healthy controls (HCs group). Compared to the late recovery stage (LRS) group, it took more than 14 days to test negative for the viral nucleic acid since clones consistently remained in the ERS while they decreased in the LRS over time. BCR has a wide range of clones that indicate the specificity of SARS-CoV-2. Consequently, B cells produce a large number of specific antibodies to suppress the
SARS-CoV-2 virus (14). This virus binds to ACE2 on the surface of spike protein (type S1) via the receptor-binding domain (RBD) and enters the host cell by activating TLR7 using endosome. Additionally, TLR7 activation stimulates the production of IFN- $\alpha$, tumor necrosis factor-alpha, IL-6, and IL-12, thereby inducing the T cytotoxic cell of $\mathrm{TCD}^{+}$and $\mathrm{T}$ helper of $\mathrm{TCD}^{+}$, as well as forming specific B-cell receptors and producing antibodies (15). In the novel coronavirus disease, peripheral lymphocytes decrease while IL-6 and C-reactive protein represent an increase (16). All types of lymphocytes including $B$ $\left(\mathrm{CD} 19^{+}\right)$, $\mathrm{T}$ (i.e., $\mathrm{CD}^{+}, \mathrm{CD}^{+}$and $\left.\mathrm{CD} 8^{+}\right)$, and natural killer (i.e., $\mathrm{CD}^{+} 6^{+}$and $\mathrm{CD} 56$ ) cells reduce in severe cases of the disease although they are close to normal status (9) and increase after treatment with corticosteroids and antivirals in normal conditions. However, post-treatment reactions vary depending on age, gender, the severity of the disease, oxygen inhalation, treatment with antivirals and corticosteroids, along with treatment with immune system boosters (17). Among lymphocytes, B cell differs significantly between COVID-19 and non-COVID-19 pneumonia patients. There is less $\mathrm{CD} 19^{+} \mathrm{B}$ cell in COVID19-induced pneumonia compared to non-COVID-19induced pneumonia. In addition, the inability of B cells may interfere with limiting the viral expansion and the spread of the virus into the cell that causes infection (18). Based on the findings of a study, B, CD19+, and $\mathrm{CD}^{+}$ cells decreased in COVID-19 patients in both normal and severe forms of the disease (19). Furthermore, the increased neutrophil and decreased lymphocyte counts led to an increase in NLR in the severe COVID-19 group. NLR is a known marker of inflammation and systemic infections that has been studied as a predictor of bacterial pneumonia infections. Moreover, increased NLR in infected patients was associated with its severity (20).

Antibody Classes and Their Specification in COVID-19 The coronavirus stimulates the host immune system when it enters the body, and then antibodies are produced and secreted to block and neutralize the virus (21). Neutralizing antibodies or Igs include Immunoglobulins $\mathrm{G}(\operatorname{IgG}), \mathrm{M}(\operatorname{Ig} \mathrm{M}), \mathrm{A}(\operatorname{Ig} \mathrm{A})$, and $\mathrm{E}$ ( $\operatorname{IgE})$ which perform antiviral operations. Additionally, IgG and $\operatorname{IgA}$ are secretory mucosal antibodies that play a significant role in the development of respiratory infections (22). Similarly, SARS-CoV-2 mostly acts like other respiratory viruses and produces secretory IgA, which is an effective protector in asymptomatic infections (23). Likewise, IgM found in the blood and lymph fluids is the first known antibody against the novel coronavirus infection. $\mathrm{IgG}$ is also the most abundant type of antibody that remains longer in these patients (24). A strong humoral response to SARS-CoV-2 is associated with the severity of the disease because evidence suggests that a strong IgG response contributes to killing the virus and may cause tissue damage due to strong immunity (25). 


\section{Antibody Production Schedule in COVID-19}

The strength of the antibody response depends on various factors such as age, nutritional status, the severity of the disease, the intake of some medications, or infections such as human immunodeficiency virus (HIV) which suppress the immune system (26). IgM is the first response, which leads to the elimination of the pathogen, and an indicator of the acute phase of the infection whereas IgG is the strongest produced antibody and an indicator of the chronic stage of the disease (27). In an SC-BCR-Seq analysis, it was found that the IgA isotype was higher in COVID-19 patients compared to the control group, followed by observing increased serum $\operatorname{IgA}$ in other coronavirus infections. Moreover, the ratio of "IgG + IgE + IgA" to "IgM + IgD" significantly increased in ERS patients and followed a decreasing trend based on recovery continuity (14). In a study on the serum of patients with COVID-19, it was revealed that IgM and $\operatorname{IgA}$ were detected in the blood of the infected patients 5 (3-6) and 14 (10-18) days after the onset of the symptoms, respectively. The level of IgM increased within days 8-14 while no further increase was observed between days 15 and 21 or after day 21. IgA antibody levels increased between days 8 and 14 whereas no further increase was found afterward. In addition, IgG levels increased during days 8-14 and 15-21, and finally, stabilized on day 21 (3). Given that NLR linked to innate immunity and IgG response associated with acquired immunity were observed in patients' immune responses, patients are classified into 4 phenotypes accordingly, including $N L R^{h i} I g G^{h i} / N L R^{h i} I g G^{l o}$ Those with the severe and deceased forms of the disease were often of the $N L R^{h i} I g G^{h i} / N L R^{h i} I g G^{l o}$ enotype. Therefore, IgG and NLR responses can be applied as a complementary tool for differentiating between severe and non-severe COVID-19 patients and thereby predicting their clinical outcomes. Further, $1.3 \%$ of patients with the $N L R^{l o} I g G^{h i}$ enotype developed a critical disease, indicating that the IgG response alone can exacerbate the disease (25). In another study on COVID-19 patients, in which the course of IgM and IgG positivity was investigated over 5 weeks, the results demonstrated that all patients tested for antibodies had symptoms after 2 weeks except for 2 patients. In the third week, all patients tested positive for IgM and IgG after developing initial symptoms. $\operatorname{IgM}$ and $\operatorname{IgG}$ were positive in all patients in the fourth week. However, IgM and IgG represented a decrease and an increase, respectively. In the fifth week, all patients tested positive for IgG whereas 2 patients tested negative for IgM (28). Based on the findings of a similar study on 6 pregnant women with COVID-19, all 6 mothers had mild clinical manifestations and all mothers gave birth in negative pressure isolation rooms in their third trimester. The obtained result from the neonates' reverse transcriptionpolymerase chain reaction (RT-PCR) was negative although serum antibodies were detected in them.
Furthermore, the concentration of $\operatorname{IgG}$ and $\operatorname{IgM}$ was higher than normal in 2 neonates and their mothers also had high IgG and IgM. Furthermore, IgG and IgM were high and normal in 3 neonates, respectively, and all mothers had high IgG and 2 of them had high IgM. Moreover, IgG increased in 5 infants and was passively transferred from the mother to the fetus via the placenta, starting in the late third trimester and rising high at birth. Additionally, the detected IgM in 2 infants was not usually transferred from the mother to the fetus due to its macromolecular structure, implying that IgM was probably produced by the infant itself (29). In SARS$\mathrm{CoV}-2$, IgM is generally produced and then switches to the production of IgG. However, IgG and IgM mostly grow at the same time range in SARS-CoV (30). More precisely, the pattern of acute viral infection is such that the concentration of IgG increases with a decrease in the levels of IgM (31). In one study, the levels of IgG and IgM antibodies against $\mathrm{N}$ and RBD proteins were observed in most patients 10 days after the onset of the symptoms. For both IgG and IgM antibodies, there was Anti RBD > Anti N. On the other hand, anti-RBD IgG levels were lower among patients with symptoms (32). The results of a study showed that the positive levels of serum IgM (S-IgM), serum IgG (S-IgG), naturally occurring IgG $(\mathrm{N}-\mathrm{IgG})$, and naturally occurring $\operatorname{IgM}(\mathrm{N}-\operatorname{IgM})$ in nonintensive care unit (non-ICU) patients gradually increased within 1 to 3 week(s) after the onset of the symptoms. In the second week, S-IgM and N-IgM peaked whereas N-IgG and S-IgG were still increasing in the third week. In this study, most ICU patients had higher N-IgG compared to S-IgG after the onset of the symptoms, probably due to the prolonged presence of the virus or exposure to large amounts of the virus. It should be pointed out that ICU patients tested positive for RNA over 31 days while non-ICU patients tested positive for RNA over 13 days. Therefore, increased N-IgG indicates the progression of the disease to severe cases. In contrast, S-IgG increased slowly in ICU patients, thus S-IgG decreased significantly in ICU patients 2 weeks after the onset of the symptoms compared with non-ICU patients, which could be a justifying reason for the prolongation of the disease in ICU patients (33). IgG levels in the severe form appear to be higher in female patients than in males. The obtained results from a similar study revealed that IgG antibody levels were higher in female patients at 2 to 4 weeks after the onset of the disease in comparison to males. This difference disappeared 4 weeks after the onset of the disease. In addition, the mortality rate was higher in men than women (34). On days after the onset of the disease symptoms, IgG responses against $\mathrm{S} 1$ and $\mathrm{N}$ increased over time. Further, another study found that IgG responses to $\mathrm{N}$ and $\mathrm{S} 1$ proteins were associated with patients' age. In male patients over 40 years of age, the produced IgG against protein S1 was extremely weakly associated with age while, in the case of women over 40 
years, IgG response to S1 was significantly higher than men and those women younger than 40 years of age. There was also no relationship between age and IgG response in men and women younger than 40 years. It was reported that $\mathrm{IgG}$ response to protein $\mathrm{S} 1, \mathrm{~N}$ was associated with lactic dehydrogenase levels in women. However, it was not the case for men. Since lactate dehydrogenase (LDH) levels can be considered as an indicator of the severity of the disease, the female immune system appears to be more sensitive to the virus. It was also revealed that the IgG response to protein $\mathrm{S} 1, \mathrm{~N}$ was negatively related to the lymphocyte percentage. Consequently, IgG is positively associated with age and $\mathrm{LDH}$ levels while negatively related to lymphocyte percentage. As regards elderly patients, a higher mortality rate belongs to men cases as compared to women since female patients produce a more effective humoral response than men (35). Differences in personal characteristics are most likely associated with inherent antibody traits and differences in patients' blood concentrations. Furthermore, the level of these antibodies is lower in individuals under 18 years of age in comparison to those over 18 years (36). Moreover, mortality rates are higher in the elderly with COVID-19, which can be explained by increased inflammatory responses and decreased antiviral cytokines in these groups of people (37). In one study, there were some reports on changes due to COVID-19 in people who died and those with a normal form of the disease. Based on the results, IgM levels were positively correlated with IgG and leukocyte count while negatively correlated with the clinical outcome and albumin level (38). In another study, the IgG level was not correlated with neutrophil and lymphocyte percentage in severe and normal groups; whereas IgM was weakly associated with the neutrophil percentage in severe patients. Therefore, IgM can be considered as an indicator of severe inflammation during acute infection (39). The findings of a similar study on an individual with COVID-19 demonstrated that the patient tested positive for HIV-1 as well. Additionally, test results revealed that the patient tested negative for RNA while positive for antibodies. Some HIV-1 drugs may act both prophylactically and therapeutically against SARS-CoV-2. Likewise, there is another possibility that the active form of IFN-1 may contribute to suppressing SARS-CoV-2. In addition, HIV1 infection may produce high levels of IFN-1, which can partially clear the infection and lead to a negative result (40). Evaluations indicated that patients whose initial RTPCR was negative tested positive for IgM and represented a positive result at the second stage of RT-PCR, indicating that antibodies can be detected to achieve the early detection of COVID-19. This, in turn, contributes to identifying the virus at this stage of the epidemic where the proper diagnosis is necessary for limiting the spread of the virus. The duration of antibody emergence is influenced by factors such as the time of sample collection and the time of symptoms onset in each particular patient (41). Negative serological and positive molecular test results imply that although individuals are infected, they have not yet reached the phase of antibody production. Conversely, those who test positive on the serological test and negative on the molecular test may be at the recovery COVID-19 phase or it may be due to inappropriate sample collection. As the antibody level rises, the viral load demonstrates a decrease. Further, the emergence of symptoms is associated with the increased sensitivity of serological tests compared to molecular tests (4). Moreover, antibodies can be produced in most infected patients except for some immunocompromised patients who are unable to effectively produce antibodies. Furthermore, there are some false positives caused by the interference of developing other diseases such as autoimmune diseases and other infections (42). False negatives have the potential to infect more individuals while false positives can test for other infections and confirm the infection as well (43).

\section{Production Role of Antibodies in COVID-19}

Protection against SARS-CoV-2 is similar to SARS$\mathrm{COV}$ in several aspects. Antibodies such as neutralizing antibodies )NAbs (and IgGs, which are produced by B cells, can block virus entry and prevent its re-spread (44). Based on the evidence, SARS-CoV-2 binds to the ACE2 receptor in respiratory epithelial cells via protein $S$ and enters the host cell. Antibodies prevent the binding of RBD (part of the $\mathrm{S} 1$ protein) to ACE2, thereby eliminating the virus infection. Additionally, antibodies targeting S14P5 and S21P2 peptides play a significant neutralizing role against the SARS-CoV-2 virus (45). In addition to RBD, S2 may be targeted by Nabs as well. Improved elderly and middleaged patients were reported to produce higher levels of antibodies against S1/RBD/S2 than younger patients although there was no difference between the patient's age and the length of hospital stay, confirming that high levels of NAb are useful for viral clearness and elderly patient recovery. NAbs are considered to be an effective way of treating or preventing the spread of viral infections. Although SARS-CoV and SARS-CoV-2 are $77.2 \%$ similar in amino acids in their spike proteins, SARS-CoV NAbs can neutralize SARS-CoV-2. On the other hand, the plasma of COVID-19 patients cannot neutralize SARS$\mathrm{CoV}$, indicating that the antigens of SARS-CoV-2 differ from those of SARS-COV (46). Antibody production is likely to lead to the formation of immune complexes with viral antigens in the alveoli of the patient's lungs. These immune complexes play a role in activating lung damage by activating neutrophils. Macrophages also stimulate severe respiratory inflammatory reactions by the phagocytosis of immune complexes via the Fc receptor, resulting in respiratory failure. In addition, IgG immune complexes in the lower respiratory tract lead to the polarization of macrophages into type 2 macrophages, as well as the 
production of IL-10, the expression of programmed death-ligand 1 , and the stimulation of regulatory $\mathrm{T}$ cells $\left(\mathrm{T}_{\text {reg }}\right.$ cells). In the fragile and anatomical environment of the lung, immune complexes and the recruitment of polymorphonucleases through an uncontrolled activation can lead to vascular endothelial damage, and translational cascade in the failure of multiple organs with thromboembolic disorders leads to death (23). One study found that recently recovered COVID-19 patients can be a good convalescent plasma donor, which may contribute to treatment. If convalescent plasma contains IgM, it means that there is a recent infection and thus may be unsuitable for donation to patients (47). Although high IgG may be effective in neutralizing COVID-19 during infection, it increases the risk of developing antibodydependent enhancement (ADE). The emergence of multiple antibodies during primary infection, after vaccination, or due to a previous infection may increase the risk of developing ADE. ADE is also associated with the development of cytokine storms. Therefore, the lack of a high affinity for IgG can reduce the severity of the infection and explain the mild disease in children compared to adults (48). Further, ADE is a phenomenon in which viral binding to non-neutralizing antibodies causes the virus to enter the host cell and proliferate occasionally. More precisely, the virus can enter the cells with no viral receptor by inducing antibodies and infect them accordingly. Furthermore, ADE can interfere with vaccine efficacy since the vaccine may produce antibodies that cause the disease to be exacerbated through ADE. It should be noted that a vaccine has been developed to protect against it (49).

\section{Antibody Duration After COVID-19 Infection}

The long-term coexistence of IgG with SARS-CoV-2 in the body raises the question of whether patients with antibodies are still at the risk of re-infection with the virus.

It is probable that IgG remains positive after the viral RNA test provides a negative result. Moreover, it causes serological tools to better help in understanding the extent of asymptomatic infections (50). It is noteworthy that antibodies produced by patients with COVID-19 may provide immunity after recovery (51). According to immunofluorescence and enzyme-linked immunosorbent assay (ELISA) measurements, specific neutralizing antibodies ( $\mathrm{IgG}$ ) were detected in $89 \%$ of recovered patients up to 2 years after SARS infection. During the long-term follow-ups of survivors, IgG can be detected in recovered patients only 6 years after SARS infection, indicating a decrease in the memory level of $\mathrm{B}$ cells, which may also be the case for SARS-CoV-2 (52). Additionally, IgG memory cells against viral RBD have been identified in the blood of COVID-19 patients, which contributes to the development of immunity after COVID-19. A new study on SARS-CoV-2 infection in macaques revealed that after the primary infection was eliminated, there was resistance to re-infection after 28 days. Nonetheless, the nature of the extent of long-term memory responses is still unknown considering the outbreak time of the disease. The lifespan of the humoral response following the SARSCoV-2 infection is also relatively short. In SARS-CoV-2, IgG against protein $S$ could be detected in the serum 60 days after the onset of the symptoms. However, the IgG titer decreased 8 weeks after the onset of the symptoms. These data suggest that the immunity of SARS-CoV-2 may reduce following an early infection, and thus further studies are required to ensure long-term protection (13). Likewise, it is unknown whether antibodies remain stable after infection in SARS-CoV-2. Even if the stable amounts of antibodies are likely to protect against re-infection, at present, it is unknown whether antibodies provide complete protection against re-infection with the same virus or a mild form of the disease is developed in case of the occurrence of re-infection with the same virus (53).

\section{Seroepidemiological Studies on COVID-19}

The prevalence of antibodies in the population seems to be trivial since the source of the virus is new. Therefore, investigating the level of antibody positivity in a population can provide information about the rate of infection and the cumulative prevalence of infection in the population. Accordingly, further extensive studies are required in this regard (54).

\section{Serological Test of COVID-19}

Multiple assays may be required to confirm the positive result of the COVID-19 antibody. In addition, serum samples should be screened for the presence of specific antibodies to the COVID-19 virus using the serological test. Tests for IgG, IgM, and IgA or total antibodies are available in this respect. Thus, the detection of total or IgG antibodies should be prioritized based on the existing evidence regarding performance. Serological tests are performed using ELISA, immunofluorescence assay, or in the case of limited laboratory capacity, the rapid diagnostic test and automated chemiluminescent immunoassay (54). To this end, serum samples are taken from patients to measure anti-SARS-CoV-2 IgM and IgG levels. All collected samples are stored at $56^{\circ} \mathrm{C}$ for 30 minutes to be inactivated (i.e., be no longer pathogenic) and then stored at $-20^{\circ} \mathrm{C}$ before testing. In some cases, the magnetic chemiluminescence enzyme immunoassay, which is based on double-antibodies sandwich immunoassay, is used to test anti-SARS-CoV-2 IgM and IgG levels. The recombinant antigen comprises the nucleoprotein and peptide of the spike protein of the SARS-CoV-2 virus conjugated to fluorescein isothiocyanate (FITC), which is stabilized on the magnetic components conjugated with the anti-FITC antibody. Further, the alkaline phosphatase test, which conjugates human IgM and IgG antibodies, is applied in antibody diagnosis. The test is automatically conducted on a magnetic chemiluminescence analyzer. 
All the above-mentioned tests are implemented under absolute biosafe conditions, and the levels of antibodies are assessed using the chemiluminescence signal (55). Experiments on several confirmed and suspected cases of COVID-19 at a center in Wuhan (China) showed that out of 66 confirmed cases, sensitivity, specificity, positive predictive value, negative predictive value, and consistency rate for IgM and IgG were $77.3 \%$ (66.51), 100\%, 100\%, $80.0 \%$, and $88.1 \%$, as well as $88.3 \%$ (66.55), $95.0 \%, 94.8 \%$, $83.8 \%$, and $88.9 \%$, respectively. Furthermore, out of 24 suspected cases, the above-mentioned measures for IgM and $\operatorname{IgG}$ were reported to be $87.5 \%$ (24.21), 100\%, 100\%, $95.2 \%$, and $96.4 \%$, as well as $70.8 \%$ (24.17), $96.6 \%, 85.0 \%$, $89.1 \%$, and $88.1 \%$, respectively (56). IgM is more sensitive to spike protein than nucleocapsid protein in the ELISA technique because protein $S$ is a transport protein that may stimulate the production of IgM antibody, especially during early infection (57). Moreover, the IgM/IgG test kit is manufactured using the gold immunochromatographic assay. It is most probable that this test kit can eliminate some of the false negatives of swab samples and can be applied as an adjunct to the RT-PCR (43). In the rapid antibody detection kit, there are 3 detection lines, among which is the control line. This detection line appears to be an example of the mainstream. The presence of antivirus $\mathrm{IgG} / \mathrm{IgM}$ is indicated by a red/pink line in the M/G region. The sample is negative if only the control line turns red while both antibodies are positive if both $G$ and $\mathrm{M}$ lines turn red. On the other hand, if the control line does not appear, the test is invalid and needs repetition. Additionally, a false-negative result is obtained if the antibody levels are less than the detection limit. In addition, differences in the production of individual antibodies can be regarded as another reason for false negatives. Antibody detection kits have several advantages over RT-PCRs. For instance, they are time-saving techniques, require no special equipment, are simple to apply, require minimal training, and can use fingertip blood instead of venous blood. Given that asymptomatic patients can spread the virus and there is no available method to differentiate asymptomatic carriers, the capability to perform this screening test for asymptomatic patients is another application of such tests (24). Based on the findings of a study, molecular tests provide more positive results in the early stages of infection whereas serological tests perform better in the later stages of infection (4). Although the IgG/IgM test is an important indicator of COVID-19 diagnosis, limitations such as changing the method and antigens of the kit are still essential for the sensitivity and characteristics of the test. Furthermore, false-positive results may be provided in case of the occurrence of crossreaction with other coronaviruses (50).

\section{Conclusion}

This study reviewed humoral immune responses and the antibody production program in patients with COVID-19.
Accordingly, further extensive research is required given that the identification of individuals suspected of having SARS-CoV-2 infection is of great importance in terms of controlling the prevalence of the disease and may help in determining the immune stability due to antibodies after recovery. It can also develop a broader vision of vaccines stimulating the humoral immune system.

\section{Conflict of Interest Disclosures}

The authors declare that they have no conflict of interests.

\section{Acknowledgements}

The authors would like to thank the Student Research Committee of Hormozgan University of Medical Sciences for their help and support.

\section{Ethical Statement}

Not applicable.

\section{Author's Contribution}

Study concept and design: ASA and ARN;Acquisition of data:FMZ and SK and MAV; Analysis and interpretation of data: ASA and FM; Manuscript drafting: ASA and FM; Critical revision of the manuscript for important intellectual content: ASA Study supervision: ASA and ARN.

\section{Funding/Support}

There is no funding for this article.

\section{Informed Consent}

Not applicable.

\section{References}

1. World Health Organization (WHO). Coronavirus Disease (COVID-2019) Situation Reports. WHO; 2020.

2. Mason RJ. Pathogenesis of COVID-19 from a cell biology perspective. Eur Respir J. 2020;55(4). doi: 10.1183/13993003.00607-2020.

3. Guo L, Ren L, Yang S, Xiao M, Chang D, Yang F, et al. Profiling early humoral response to diagnose novel coronavirus disease (COVID-19). Clin Infect Dis. 2020;71(15):778-85. doi: 10.1093/cid/ciaa310.

4. Paradiso AV, De Summa S, Loconsole D, Procacci V, Sallustio $A$, Centrone A, et al. Clinical meanings of rapid serological assay in patients tested for SARS-Co2 RT-PCR. medRxiv. 2020. doi: 10.1101/2020.04.03.20052183.

5. Yong G, Yi Y, Tuantuan L, Xiaowu W, Xiuyong L, Ang L, et al. Evaluation of the auxiliary diagnostic value of antibody assays for the detection of novel coronavirus (SARS-CoV-2). J Med Virol. 2020. doi: 10.1002/jmv.25919.

6. Jin Y, Yang H, Ji W, Wu W, Chen S, Zhang W, et al. Virology, epidemiology, pathogenesis, and control of COVID-19. Viruses. 2020;12(4). doi: 10.3390/v12040372.

7. Li G, Fan Y, LaiY, Han T, Li Z, Zhou P, etal. Coronavirus infections and immune responses. J Med Virol. 2020;92(4):424-32. doi: 10.1002/jmv.25685.

8. Wilk CM. Coronaviruses hijack the complement system. Nat Rev Immunol. 2020;20(6):350. doi: 10.1038/s41577-0200314-5.

9. He R, Lu Z, Zhang L, Fan T, Xiong R, Shen X, et al. The clinical course and its correlated immune status in COVID-19 pneumonia. J Clin Virol. 2020;127:104361. doi: 10.1016/j. 


\section{jcv.2020.104361.}

10. Zamani P, Gholizadeh Navashenaq J, Nikpoor AR, Hatamipour M, Kazemi Oskuee R, Badiee A, et al. MPL nanoliposomal vaccine containing P5 HER2/neu-derived peptide pulsed PADRE as an effective vaccine in a mice TUBO model of breast cancer. J Control Release. 2019;303:223-36. doi: 10.1016/j.jconrel.2019.04.019.

11. Barati N, Nikpoor AR, Razazan A, Mosaffa F, Badiee A, Arab A, et al. Nanoliposomes carrying HER2/neu-derived peptide AE36 with CpG-ODN exhibit therapeutic and prophylactic activities in a mice TUBO model of breast cancer. Immunol Lett. 2017;190:108-17. doi: 10.1016/j.imlet.2017.07.009.

12. Nikpoor AR, Jaafari MR, Zamani P, Teymouri M, Gouklani $H$, Saburi E, et al. Cell cytotoxicity, immunostimulatory and antitumor effects of lipid content of liposomal delivery platforms in cancer immunotherapies. A comprehensive invivo and in-vitro study. Int J Pharm. 2019;567:118492. doi: 10.1016/j.ijpharm.2019.118492.

13. Vabret N, Britton GJ, Gruber C, Hegde S, Kim J, Kuksin $M$, et al. Immunology of COVID-19: current state of the science. Immunity. 2020;52(6):910-41. doi: 10.1016/j. immuni.2020.05.002.

14. Wen W, Su W, Tang H, Le W, Zhang X, Zheng Y, et al. Immune cell profiling of COVID-19 patients in the recovery stage by single-cell sequencing. Cell Discov. 2020;6:31. doi: 10.1038/ s41421-020-0168-9.

15. Ahmadpoor P, Rostaing L. Why the immune system fails to mount an adaptive immune response to a COVID-19 infection. Transpl Int. 2020;33(7):824-5. doi: 10.1111/tri.13611.

16. Ma J, Xia P, Zhou Y, Liu Z, Zhou X, Wang J, et al. Potential effect of blood purification therapy in reducing cytokine storm as a late complication of critically ill COVID-19. Clin Immunol. 2020;214:108408. doi: 10.1016/j.clim.2020.108408.

17. Wang $F$, Nie J, Wang $H$, Zhao Q, Xiong $Y$, Deng L, et al. Characteristics of peripheral lymphocyte subset alteration in COVID-19 pneumonia. J Infect Dis. 2020;221(11):1762-9. doi: 10.1093/infdis/jiaa150.

18. Zheng Y, Huang Z, Ying G, Zhang X, Ye W, Hu Z, et al. Study of the lymphocyte change between COVID-19 and nonCOVID-19 pneumonia cases suggesting other factors besides uncontrolled inflammation contributed to multi-organ injury. medRxiv. 2020. doi: 10.1101/2020.02.19.20024885.

19. Tan M, Liu Y, Zhou R, Deng X, Li F, Liang K, et al. Immunopathological characteristics of coronavirus disease 2019 cases in Guangzhou, China. Immunology. 2020;160(3):261-8. doi: 10.1111/imm.13223.

20. Qin C, Zhou L, Hu Z, Zhang S, Yang S, Tao Y, et al. Dysregulation of immune response in patients with coronavirus 2019 (COVID-19) in Wuhan, China. Clin Infect Dis. 2020;71(15):762-8. doi: 10.1093/cid/ciaa248.

21. Zhou G, Zhao Q. Perspectives on therapeutic neutralizing antibodies against the Novel Coronavirus SARS-CoV-2. Int J Biol Sci. 2020;16(10):1718-23. doi: 10.7150/ijbs.45123.

22. Mohamed AA, Alawna M. Role of increasing the aerobic capacity on improving the function of immune and respiratory systems in patients with coronavirus (COVID-19): a review. Diabetes Metab Syndr. 2020;14(4):489-96. doi: 10.1016/j. dsx.2020.04.038.

23. Béné MC, de Carvalho Bittencourt M, Eveillard M, Le Bris Y. Good IgA bad IgG in SARS-CoV-2 infection? Clin Infect Dis. 2020;71(15):897-8. doi: 10.1093/cid/ciaa426.

24. Li Z, Yi Y, Luo X, Xiong N, Liu Y, Li S, et al. Development and clinical application of a rapid IgM-IgG combined antibody test for SARS-CoV-2 infection diagnosis. J Med Virol. 2020. doi: 10.1002/jmv.25727.

25. Zhang B, Zhou X, Zhu C, Song Y, Feng F, Qiu Y, et al. Immune phenotyping based on the neutrophil-to-lymphocyte ratio and IgG level predicts disease severity and outcome for patients with COVID-19. Front Mol Biosci. 2020;7:157. doi: 10.3389/ fmolb.2020.00157.

26. World Health Organization (WHO). Advice on the Use of Point-of-Care Immunodiagnostic Tests for COVID-19: Scientific Brief, 8 April 2020. WHO; 2020.

27. Pan Y, Li X, Yang G, Fan J, Tang Y, Zhao J, et al. Serological immunochromatographic approach in diagnosis with SARS-CoV-2 infected COVID-19 patients. J Infect. 2020;81(1):e28-e32. doi: 10.1016/j.jinf.2020.03.051.

28. Xiao AT, Gao C, Zhang S. Profile of specific antibodies to SARS-CoV-2: The first report. J Infect. 2020;81(1):147-78. doi: 10.1016/j.jinf.2020.03.012.

29. Zeng H, Xu C, Fan J, Tang Y, Deng Q, Zhang W, et al. Antibodies in infants born to mothers with COVID-19 pneumonia. JAMA. 2020;323(18):1848-9. doi: 10.1001/jama.2020.4861.

30. Hoffman T, Nissen K, Krambrich J, Rönnberg B, Akaberi D, Esmaeilzadeh M, et al. Evaluation of a COVID-19 IgM and IgG rapid test; an efficient tool for assessment of past exposure to SARS-CoV-2. Infect Ecol Epidemiol. 2020;10(1):1754538. doi: 10.1080/20008686.2020.1754538.

31. Liu W, Liu L, Kou G, Zheng Y, Ding Y, Ni W, et al. Evaluation of nucleocapsid and spike protein-based enzyme-linked immunosorbent assays for detecting antibodies against SARS-CoV-2. J Clin Microbiol. 2020;58(6). doi: 10.1128/ jcm.00461-20.

32. To KK, Tsang OT, Leung WS, Tam AR, Wu TC, Lung DC, et al. Temporal profiles of viral load in posterior oropharyngeal saliva samples and serum antibody responses during infection by SARS-CoV-2: an observational cohort study. Lancet Infect Dis. 2020;20(5):565-74. doi: 10.1016/s1473-3099(20)301961.

33. Sun B, Feng $Y$, Mo X, Zheng P, Wang Q, Li P, et al. Kinetics of SARS-CoV-2 specific IgM and IgG responses in COVID-19 patients. Emerg Microbes Infect. 2020;9(1):940-8. doi: 10.1080/22221751.2020.1762515.

34. Zeng F, Dai C, Cai P, Wang J, Xu L, Li J, et al. A comparison study of SARS-CoV-2 IgG antibody between male and female COVID-19 patients: a possible reason underlying different outcome between sex. J Med Virol. 2020. doi: 10.1002/ jmv.25989.

35. Jiang $H$, Li $Y$, Zhang $H$, Wang $W$, Men $D$, Yang $X$, et al. Global profiling of SARS-CoV-2 specific IgG/lgM responses of convalescents using a proteome microarray. medRxiv. 2020. doi: 10.1101/2020.03.20.20039495.

36. Lin D, Liu L, Zhang M, HuY, Yang Q, Guo J, et al. Evaluations of the serological test in the diagnosis of 2019 novel coronavirus (SARS-CoV-2) infections during the COVID-19 outbreak. medrxiv. 2020. doi: 10.1101/2020.03.27.20045153.

37. Wang B, Wang L, Kong X, Geng J, Xiao D, Ma C, et al. Longterm coexistence of SARS-CoV-2 with antibody response in COVID-19 patients. J Med Virol. 2020. doi: 10.1002/ jmv.25946.

38. Wang Z, Li H, Li J, Yang C, Guo X, Hu Z, et al. Elevated serum IgM levels indicate poor outcome in patients with coronavirus disease 2019 pneumonia: A retrospective case-control study. medRxiv. 2020. doi: 10.1101/2020.03.22.20041285.

39. Xie J, Ding C, Li J, Wang Y, Guo H, Lu Z, et al. Characteristics of patients with coronavirus disease (COVID-19) confirmed 
using an IgM-IgG antibody test. J Med Virol. 2020. doi: 10.1002/jmv.25930.

40. Zhao J, Liao X, Wang H, Wei L, Xing M, Liu L, et al. Early virus clearance and delayed antibody response in a case of COVID-19 with a history of co-infection with HIV-1 and HCV. Clin Infect Dis. 2020. doi: 10.1093/cid/ciaa408.

41. Zhang J, Zhang X, Liu J, Ban Y, Li N, Wu Y, et al. Serological detection of 2019-nCoV respond to the epidemic: a useful complement to nucleic acid testing. Int Immunopharmacol. 2020;88:106861. doi: 10.1016/j.intimp.2020.106861.

42. Yangchun F. Optimize clinical laboratory diagnosis of COVID-19 from suspect cases by likelihood ratio of SARS-CoV-2 IgM and IgG antibody. medRxiv. 2020. doi: 10.1101/2020.04.07.20053660.

43. Liu Y, Liu Y, Diao B, Ren F, Wang Y, Ding J, et al. Diagnostic indexes of a rapid IgG/IgM combined antibody test for SARSCoV-2. medRxiv. 2020. doi: 10.1101/2020.03.26.20044883.

44. Lin Q, Zhu L, Ni Z, Meng H, You L. Duration of serum neutralizing antibodies for SARS-CoV-2: lessons from SARSCoV infection. J Microbiol Immunol Infect. 2020;53(5):821-2. doi: 10.1016/j.jmii.2020.03.015.

45. Poh CM, Carissimo G, Wang B, Amrun SN, Lee CYP, Chee RSL, et al. Potent neutralizing antibodies in the sera of convalescent COVID-19 patients are directed against conserved linear epitopes on the SARS-CoV-2 spike protein. bioRxiv. 2020. doi: 10.1101/2020.03.30.015461.

46. Wu F, Wang A, Liu M, Wang Q, Chen J, Xia S, et al. Neutralizing antibody responses to SARS-CoV-2 in a COVID-19 recovered patient cohort and their implications. medRxiv. 2020. doi: 10.1101/2020.03.30.20047365.

47. Zhang L, Pang R, Xue X, Bao J, Ye S, Dai Y, et al. Anti-SARSCoV-2 virus antibody levels in convalescent plasma of six donors who have recovered from COVID-19. Aging (Albany NY). 2020;12(8):6536-42. doi: 10.18632/aging.103102.

48. Wang J, Zand MS. The potential for antibody-dependent enhancement of SARS-CoV-2 infection: translational implications for vaccine development. J Clin Transl Sci. 2020. doi: $10.1017 /$ cts.2020.39

49. Peron JPS, Nakaya H. Susceptibility of the Elderly to SARSCoV-2 Infection: ACE-2 Overexpression, Shedding, and Antibody-dependent Enhancement (ADE). Clinics (Sao Paulo). 2020;75:e1912. doi: 10.6061/clinics/2020/e1912.

50. Gao HX, Li YN, Xu ZG, Wang YL, Wang HB, Cao JF, et al. Detection of serum immunoglobulin $\mathrm{M}$ and immunoglobulin $G$ antibodies in 2019 novel coronavirus infected patients from different stages. Chin Med J (Engl). 2020;133(12):1479-80. doi: $10.1097 / \mathrm{cm} 9.0000000000000820$.

51. Du Z, Zhu F, Guo F, Yang B, Wang T. Detection of antibodies against SARS-CoV-2 in patients with COVID-19. J Med Virol. 2020. doi: 10.1002/jmv.25820.

52. Rokni M, Ghasemi V, Tavakoli Z. Immune responses and pathogenesis of SARS-CoV-2 during an outbreak in Iran: comparison with SARS and MERS. Rev Med Virol. 2020;30(3):e2107. doi: 10.1002/rmv.2107.

53. Flodgren GM. Immunity After SARS-CoV-2 Infection, 1st Update-A Rapid Review. Oslo: Norwegian Institute of Public Health; 2020

54. World Health Organization (WHO). Population-Based Age-Stratified Seroepidemiological Investigation Protocol for Coronavirus 2019 ('COVID-19)| Infection, 26 May 2020, version 2.0. WHO; 2020.

55. Long QX, Liu BZ, Deng HJ, Wu GC, Deng K, Chen YK, et al. Antibody responses to SARS-CoV-2 in patients with COVID-19. Nat Med. 2020;26(6):845-8. doi: 10.1038/ s41591-020-0897-1.

56. Xiang F, Wang X, He X, Peng Z, Yang B, Zhang J, et al. Antibody detection and dynamic characteristics in patients with COVID-19. Clin Infect Dis. 2020. doi: 10.1093/cid/ ciaa461.

57. Zhong L, Chuan J, Gong B, Shuai P, Zhou Y, Zhang Y, et al. Detection of serum $\lg M$ and $\lg G$ for COVID-19 diagnosis. Sci China Life Sci. 2020;63(5):777-80. doi: 10.1007/s11427-0201688-9. 